\title{
Роль витаминов
}

\section{и микроэлементов \\ в парентера^ьном питании новоро»кАенНых}

\author{
Горелик K.A. ${ }^{1}$, \\ Горелик Ю.В. ${ }^{2}$, \\ Амитриев А.B. ${ }^{3}$, \\ Быков К.B. ${ }^{2}$
}

\begin{abstract}
${ }^{1}$ Федеральное государственное бюджетное образовательное учреждение высшего образования "Первый Санкт-Петербургский государственный медицинский университет имени академика И.П. Павлова" Министерства зАравоохранения Российской Федерации, 197022, г. Санкт-Петербург, Российская Федерация

${ }^{2}$ Санкт-Петербургское бюджетное учреждение зАравоохранения "Аетский городской многопрофильный клинический специализированный центр высоких меАицинских технологий", 198205, г. Санкт-Петербург, Российская Федерация

${ }^{3}$ Северо-Западная ассоциация парентерального и энтерального питания, 191023, г. Санкт-Петербург, Российская Федерация
\end{abstract}

Витамины и микроэлементы являются важными компонентами для роста и развития плода в течение всего внутриутробного развития, а также у новорожденных в весь последующий период грудного вскармливания. В случае когда проведение энтерального питания невозможно или ограничено, необходимо назначение парентеральной формы данных компонентов.

В обзоре представлен анализ материалов о количественной и качественной составляющей парентерального питания новорожденных - витаминах и микроэлементах.

Финансирование. Исследование не имело спонсорской поддержки.

Конфликт интересов. Авторы заявляют об отсутствии конфликта интересов.

Для цитирования: Горелик К.Д., Горелик Ю.В., Дмитриев А.В., Быков К.В. Роль витаминов и микроэлементов в парентеральном питании новорожденных // Неонатология: новости, мнения, обучение. 2020. Т. 8, № 2. С. 39-46. D0I: 10.33029/2308-24022020-8-2-39-46

Статья поступила в редакцию 09.04.2020. Принята в печать 04.06.2020.

The role of vitamins and trace elements in newborns parenteral nutrition

Gorelik K.D. ${ }^{1}$, Gorelik Yu.V. ${ }^{2}$, Dmitriev A.V. ${ }^{3}$, Bykov K.V. ${ }^{2}$

${ }^{1}$ Pavlov First Saint Petersburg State Medical University, 197022, Saint

Petersburg, Russian Federation

${ }^{2}$ Children's City Multidisciplinary Clinical Specialized Center of High Medical Technologies, 198205, Saint Petersburg, Russian Federation

${ }^{3}$ Nord-West Association of Parenteral and Enteral Nutrition, 191023, Saint Petersburg, Russian Federation
Ключевье слова: витамины, микроэлементы, парентеральное питание, новорожденные
Vitamins and trace elements are an important component for the growth and development of the fetus during the intrauterine development as well as in newborns during of breastfeeding period. In the case when the enteral nutrition is not possible or limited, it is necessary to prescribe the parenteral form of these components. This literature review presents an analysis of materials on the quantitative and qualitative component of the parenteral nutrition of newborns - vitamins and trace elements.
Keywords:

vitamins, trace elements, parenteral nutrition, newborns 
Funding. The study had no sponsor support.

Conflict of interests. The author declare no conflict of interests.

For citation: Gorelik K.D., Gorelik Yu.V., Dmitriev A.V., Bykov K.V. The role of vitamins and trace elements in newborns parenteral nutrition. Neonatologiya: novosti, mneniya, obuchenie [Neonatology: News, Opinions, Training]. 2020; 8 (2): 39-46. D0I: 10.33029/ 2308-2402-2020-8-2-39-46 (in Russian)

Received 09.04.2020. Accepted 04.06.2020.

$\mathrm{B}$ итамины и микроэлементы являются незаменимыми веществами для роста и развития плода в течение всего внутриутробного развития, а также у новорожденных в весь последующий период грудного вскармливания. В клинических условиях, когда нет иного способа обеспечения жизнедеятельности новорожденного, кроме частичного или полного парентерального питания, адекватное снабжение белками, жирами, углеводами, минералами, витаминами и микроэлементами играет критически важную роль [1-4]. Основные положения стратегии ведения новорожденных, находящихся на парентеральном питании, отражены в рекомендациях ESPGHAN/ESPEN [5] и в клинических рекомендациях по парентеральному питанию новорожденных, подготовленных Российской ассоциацией специалистов перинатальной медицины совместно с Российским обществом неонатологов [6].

Однако неонатологи, назначая парентеральное питание, далеко не всегда учитывают необходимость дополнения внутривенных питательных смесей витаминами и микроэлементами. Как отмечено в работе Ю.В. Ерпулевой и А.У. Лекманова [1] недостаточность поступления в организм железа, цинка, меди, молибдена, витаминов A, E, B 12 и фолатов частое осложнение парентерального питания в неонатологии. Особенно значимым становится дефицит витаминов и микроэлементов при развитии осложнений длительного парентерального питания, например холестаза [2]. Даже при частичном парентеральном питании, когда сохраняется определенный объем энтерального поступления нутриентов, например при синдроме холестаза, уменьшается количество поступающей в кишечник желчи, что приводит к нарушению процессов всасывания жиров и жирорастворимых витаминов A, D, E и K. С увеличением длительности проведения парентерального питания возрастает риск развития метаболической болезни костей (МБК) - у 16-40\% недоношенных с очень низкой (ОНМТ) и экстремально низкой массой тела (ЭНMT), что требует контроля показателей обмена кальция и фосфора и поддержания рекомендуемого уровня потребления витамина D [2]. Дефицит витамина K приводит к нарушению функции печени и развитию коагулопатий.

Как известно, материнское молоко - оптимальный источник всех питательных веществ для ребенка, особенно в первые 6 мес от рождения. Несмотря на относительно невысокую концентрацию витаминов и микроэлементов в грудном молоке, они играют важную роль во всех без исключения процессах растущего организма, а их недостаточное поступление замедляет рост, вызывает анемию, снижает иммунитет и вызывает органную дисфункцию, особенно у недоношенных детей $[7,8]$. В процессе беременности концентрация витаминов и микроэлементов в крови плода отражает концентрацию этих веществ в сыворотке крови матери $[9,10]$.

\section{Уровни витаминов и микроэлементов

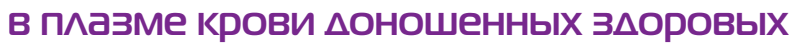 Аетей и сравнение с составом грудного молока и плазмы крови матерей}

В исследовании H.S. Özdemir и соавт. [10] проведена сравнительная оценка содержания витаминов, минералов и микроэлементов в плазме крови матери, новорожденного и в материнском молоке. Содержание ретинола, $\alpha$-токоферола и $\gamma$-токоферола в плазме крови новорожденных было достоверно ниже, чем в материнской плазме (примерно в 2-7 раз), что связано с низким содержанием этих витаминов в материнском молоке. Содержание калия и марганца в плазме крови матерей и новорожденных было примерно одинаковым, в то время как содержание железа и цинка у новорожденных было примерно на 25-30\% выше, а магния и меди - ниже примерно на 10 и 70\% соответственно. Средние значения содержания микроэлементов в плазме крови новорожденных составили (мг/л): железо - 2,38; медь - 0,32; магний - 18,02; цинк -1,22; марганец - 0,03. Уровень витамина А в пуповинной крови у здоровых младенцев варьирует от 15,7 до 20,4 мкг/дл [11]. Уровень витамина Е в плазме крови новорожденных достаточно низок (2,5 мг/л), что на 25\% ниже, чем у матерей. Однако впоследствии его содержание в плазме детей быстро растет, за исключением младенцев, находящихся на искусственном вскармливании, что предопределяет необходимость дополнительного применения витамина $\mathrm{E}$ в дозе примерно 3 МЕ/кг массы тела [12].

Рекомендованные суточные дозы поступления витаминов здоровым новорожденным представлены в табл. 1 [13].

\section{Уровни витаминов и микроэлементов у неАоношенных $\triangle$ етей (с очень нИзКой и экстремально низкой массой тела при рожАении) и при развитии патологических состояний}

Buтамин D. Поддержание адекватного уровня витамина D в плазме крови новорожденных имеет важное значение не только для развития и роста, но и для протекания патологических процессов при их возникновении. Оптимальная доза витамина D для парентерального питания (ПП) недоношенных новорожденных точно не установлена, но на практике составляет как минимум 30 ME на 1 кг массы тела в день внутривенно в составе жировых эмульсий [14].

Неонатальный сепсис у недоношенных детей остается одной из главных причин развития осложнений и летальности из-за незрелой иммунной системы [15]. Эта категория пациентов рассматривается как иммуносупрессивная, у которой каждая инфекция является серьезной 
Таблица 1. Эталонное потребление (Dietary Reference Intakes - DRIs): рекомендуемые пищевые нормы (Recommended Dietary Allowances - RDA) и адекватное потребление (Adequate Intakes - Al ) витаминов в первые 6 мес жизни (Институт медицинь национальной академии США; цит. по: [13])

\begin{tabular}{|c|c|c|c|c|c|c|c|c|c|c|c|c|c|}
\hline \multicolumn{14}{|c|}{ Рекомендуемая норма потребления витаминов в первые 6 мес жизни } \\
\hline $\begin{array}{c}\text { A, } \\
\text { MKг/ }\end{array}$ & $\begin{array}{c}\text { D, } \\
\text { мKг/ }\end{array}$ & $\begin{array}{c}\text { E, } \\
\text { мг/ }\end{array}$ & $\begin{array}{c}\mathrm{K}, \\
\text { MKг/ }\end{array}$ & $\begin{array}{c}\mathrm{C} \\
\mathrm{Mr} /\end{array}$ & $\begin{array}{l}\mathrm{B}_{1} \\
\mathrm{Mr} /\end{array}$ & $\begin{array}{l}\mathrm{B}_{2} \\
\mathrm{Mr} /\end{array}$ & $\begin{array}{l}\mathrm{B}_{3} \\
\mathrm{Mr} /\end{array}$ & $\begin{array}{l}\mathrm{B}_{6}, \\
\mathrm{Mr} /\end{array}$ & $\begin{array}{c}\mathrm{B}_{9} \\
\text { MKг/ }\end{array}$ & $\begin{array}{c}\mathrm{B}_{12} \\
\mathrm{MKr} /\end{array}$ & $\begin{array}{l}\mathrm{B}_{5}, \\
\mathrm{Mr} /\end{array}$ & $\begin{array}{c}\mathrm{B}_{7} \\
\text { MKг/ }\end{array}$ & $\begin{array}{c}\mathrm{B}_{4}, \\
\mathrm{Mr} /\end{array}$ \\
\hline Аень & день & день & Аень & Аень & Аень & Аень & Аень & Аень & Аень & Аень & день & день & Аень \\
\hline 400 & 5 & 4 & 2 & 40 & 0,2 & 0,3 & 2 & 0,1 & 65 & 0,4 & 1,7 & 5 & 125 \\
\hline
\end{tabular}

и характеризуется быстрым переходом от бактериемии к сепсису, септическому шоку с полиорганной недостаточностью и диссеминированным внутрисосудистым свертыванием (ДВС-синдромом).

В работе T.S. Gamal и соавт. [16] изучали уровни метаболита 25(OH) витамина D в сыворотке крови младенцев с начальной стадией сепсиса ( $n=50$, из них 25 доношенных и 25 недоношенных) в сравнении с группой здоровых детей аналогичного возраста. У всех детей определялись также показатели крови, С-реактивный белок, проводилось клиническое обследование. Выявлено, что новорожденные с врожденным сепсисом (Early-Onset Sepsis - EOS) имеют более низкие уровни витамина D в плазме крови: недоно-

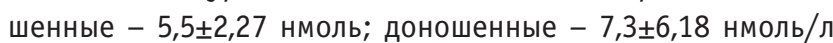
по сравнению с группой здоровых детей. Это согласуется с данными во взрослой популяции с сепсисом в отделениях реанимации и интенсивной терапии (ОРИТ), где уровень витамина D имеет отрицательную корреляционную связь с показателями летальности, а относительно высокие показатели сопровождаются уменьшением времени госпитализации $[17,18]$.

T.S. Gamal и соавт. считают, что низкий уровень витамина $D$ является фактором риска из-за важной роли иммунитета в патофизиологии сепсиса и формировании системного воспалительного ответа. Считается, что в течение всего первого года жизни детей низкий уровень витамина D accoциирован с увеличением частоты развития сепсиса [19]. В условиях дефицита витамина D могут возникать нарушения функции макрофагов и увеличиваться продукция провоспалительных цитокинов. Напротив, активация рецепторов витамина D на мембранах всех иммунных клеток стимулирует функции моноцитов, макрофагов и нейтрофилов, что ведет к усилению хемотаксиса и фагоцитоза, усиливает продукцию антимикробных пептидов (например, кателицидина), которые подавляют рост грамположительных и грамотрицательных бактерий.

Витамин E. Дети уже при рождении имеют дефицит витамина Е, что с первого дня жизни требует адекватного поступления этого витамина, которое может быть обеспечено полноценным грудным вскармливание. Если это не обеспечивается в силу тех или иных причин, возникающий дефицит может характеризоваться гемолитической анемией [20]. Американская академия педиатрии и Американское общество клинического питания рекомендует рутинное использование витамина Е в дозе 2,8-3,5 ME (максимум 7,0 ME) на 1 кг массы тела в день у новорожденных с ОНМТ при рождении [21]. Дополнительное назначение витамина Е, при котором достигается концентрация в плазме крови 3,5 мг/дл, достоверно снижает риск развития ретинопатии у недоношенных, вероятность развития внутричерепных кровоизлияний, гемолитической анемии и бронхолегочной дисплазии [22]. Кроме того, антиоксидантное действие витамина Е улучшает функцию печени, снижает уровень билирубина, обеспечивая гепатопротекторное действие. В то же время не следует превышать рекомендуемые дозы из-за возможности развития побочных эффектов.

Витамин A. У недоношенных детей дефицит витамина А играет определенную роль в развитии респираторных инфекций и бронхолегочной дисплазии. У $76 \%$ младенцев с ОНМТ имеется дефицит витамина А, проявляющийся снижением концентрации витамина А и $\beta$-каротина в плазме крови; у доношенных детей частота дефицита витамина $A$ составляет $67 \%$ [23]. У детей на ПП дефицит витамина А становится максимальным. Считается, что он возникает также при таких патологических состояниях, как сепсис, течение ВИЧ-инфекции (вирус иммунодефицита человека), ожоги, искусственная вентиляция легких, кишечная дисфункция, почечная недостаточность, нарушения питания (недостаточность поступления белка и энергии), гепатобилиарная дисфункция, травма, недостаточность цинка, и некоторых других. Концентрацию витамина А в сыворотке крови недоношенных младенцев ниже 200 мг/л (0,7 ммоль/л) рассматривают как маркер дефицита, а концентрацию ниже $100 \mathrm{mr/л} \mathrm{-} \mathrm{как} \mathrm{маркер} \mathrm{выраженного} \mathrm{дефицита} \mathrm{и} \mathrm{сниже-}$ ния запасов этого витамина в печени. В норме для детей старше 6 мес диапазон концентраций витамина А составляет 300-800 мг/л (1,05-2,8 ммоль/л). Кохрановский анализ выявил достоверную связь между уровнем обеспечения витамином А в первый месяц жизни ребенка с ОНМТ при рождении, летальностью и потребностью в дополнительной подаче $[24,25]$. Как правило, витамин A, как и другие жирорастворимые витамины, вводится в составе жировой эмульсии. Клинические исследования подтвердили повышение уровней витаминов А и Е в плазме крови новорожденных, которые получали жировые эмульсии, обогащенные $\omega$-3 жирными кислотами, что сопровождалось снижением оксидативного стресса [26].

Витамин K. Регулирует карбоксилирование факторов свертывания крови II, VII, IX и X, а также участвует в синтезе остеокальцина - маркера формирования костной ткани. В случаях выраженной недостаточности у новорожденных витамина К могут возникать кровоизлияния в желудочнокишечном тракте (ЖКТ), коже и других органах и тканях. Риск этих явлений усиливается при таких заболеваниях и условиях, как муковисцидоз, холестаз, применение антикоа- 
гулянтов, антиконвульсантов и пр. Рекомендуемая Американской академией педиатрии доза для парентеральной формы витамина К составляет 80 мкг на 1 кг массы тела в день.

Витамин С. Является кофактором многих ферментов и антиоксидантом. Системное воспаление и недостаточность питания повышают потребность в витамине С. Рекомендованная доза для парентерального питания составляет 15-25 мг на 1 кг массы тела в день.

Витамин $B_{1}$ (тиамин). Участвует в углеводном и жировом обмене, поэтому потребность в нем зависит от режима ПП (углеводный и липидный компоненты). Дефицит витамина $\mathrm{B}_{1}$ у детей, включая младенцев, на ПП может приводить к развитию лактат-ацидоза, энцефалопатии и даже смерти в течение дней или недель $[27,28]$. Рекомендованная доза при проведении ПП - 200-350 мкг/кг в день.

Витамин $B_{2}$ (рибофлавин). Участвует в энергетическом метаболизме, а его поступление связано с потреблением протеинов. Адекватность обеспечения рибофлавином может быть оценена по уровню его концентрации в плазме крови и уровню глутатион-редуктазы в эритроцитах. Дефицит рибофлавина проявляется гиперемией слизистых оболочек, развитием стоматитов и дерматитов, нарушениями зрения и анемией. Рекомендованная доза рибофлавина для недоношенных младенцев при проведении ПП составляет 0,15-0,2 мг/кг в день.

Витамин $B_{6}$ (пиридоксин). Является кофактором более 100 ферментов и участвует в процессах гликолиза, глюконеогенеза и метаболизма аминокислот. Необходим для формирования иммунитета ребенка. Дефицит пиридоксина у новорожденных проявляется в виде дерматитов, анемии, судорог, снижения иммунитета, энцефалопатии и гипергомоцистеинемии. Недоношенные младенцы, в отличие от доношенных, имеют незрелую ферментную систему, в которой участвует пиридоксин, что обусловливает неврологические нарушения, проявляющиеся нейропатией и эпилепсией [29]. Рекомендованная доза пиридоксина составляет 0,150,2 мг/кг в день.

Витамин $B_{12}$ (кобаламин). Участвует в метаболических реакциях, связанных с синтезом нуклеотидов ДНК. Риск дефицита кобаламина возникает у младенцев на ПП после резекции тонкой кишки (синдром короткой кишки), что сопровождается неврологическими и гематологическими нарушениями. Рекомендованная доза кобаламина составляет 0,6 мкг/кг в день.

Ниацин. Участвует в синтезе НАД и НАДФ, что обеспечивает транспорт электронов и энергетический метаболизм. Дефицит ниацина характеризуется неврологическими и кишечными нарушениями. Рекомендованная доза составляет для недоношенных и доношенных младенцев 4-6,8 мг/кг в день.

Пантотеновая кислота. Необходима для синтеза коэнзима А, что обусловливает ее участие в метаболизме жиров. Дефицит пантотеновой кислоты развивается достаточно редко, но его вероятность на полном ПП имеет место. Рекомендованная доза для недоношенных и доношенных младенцев составляет 2,5 мг/кг в день.

Биотин. Долгосрочное ПП без биотина на фоне длительного применения антибиотиков широкого спектра действия может приводить к летаргии, гипотензии, дерматитам, анорексии, гиперестезии, мышечным болям и ряду других проявлений. У детей с синдромом короткой кишки может возникать вторичный дефицит биотина, сопровождающийся лактат-ацидозом [30].

Фолиевая кислота. Участвует в синтезе пуринов и пиримидинов, клеточном митозе, метаболизме некоторых аминокислот, катаболизме гистидина и целом ряде других биохимических процессов. Недоношенные младенцы имеют низкий уровень фолиевой кислоты в первые 2-3 мес жизни, который не обеспечивает нормальный уровень роста и развития. Дефицит фолиевой кислоты может усиливаться при применении антибиотиков, антиконвульсантов, а также при наличии мальабсорбции в кишечнике. Это может приводить к развитию у младенцев мегалобластической анемии [31]. Рекомендованная доза для доношенных и недоношенных младенцев составляет 56 мкг/кг в день.

Микроэлементы. У недоношенных младенцев высок риск развития микроэлементной недостаточности, в частности дефицита железа, цинка и селена, в период от 2 до 6 мес от рождения [32]. В результате дефицита цинка у детей на грудном вскармливании могут возникнуть алопеция и дерматит, которые усиливаются на искусственном вскармливании. В зависимости от состава используемых молочных смесей как основного источника питания существует риск недостаточности поступления в организм цинка и меди. В исследовании [32] у $60 \%$ младенцев отмечался дефицит цинка, а пищевые добавки этого микроэлемента способствовали увеличению массы тела. Для лечения рекомендованы добавки соединений цинка 1 мг на 1 кг массы тела в день до достижения уровней цинка в плазме крови 70 мкг/дл.

Дефицит железа часто встречается у недоношенных младенцев и может проявляться в более поздних сроках после рождения (после 6-16 мес) в виде анорексии, замедления набора массы тела, бледности кожных покровов, дисфории и снижения уровней гемоглобина $\leq 100$ г/л. В качестве мер лечения дефицита железа рекомендуются добавки соединений железа и железосодержащих препаратов в состав питательных смесей в дозировке 2 мг на 1 кг массы тела в день, а при массе тела менее 1000 г - 3-4 мг/кг до нормализации уровней железа и ферритина в плазме крови [32].

У недоношенных младенцев может возникать дефицит меди и селена, который, однако, не ведет в большинстве случаев ни к каким клиническим последствиям. С другой стороны, при проведении парентерального питания этот фактор следует учитывать и проводить соответствующую коррекцию, особенно в период быстрого роста (2-6 мес после рождения).

Рекомендации международных организаций по клиническому питанию по применению витаминов и микроэлементов при проведении парентерального питания в неонатологии (ESPGHAN/ESPEN/ESPR/CSPEN) (табл. 2, 3) [33, 34]. Основные положения этих организаций, касающиеся применения витаминов и микроэлементов для парентерального питания в неонатологии, сводятся к следующему.

- Новорожденные, находящиеся на ПП, должны получать дополнительно парентеральные формы витаминов. 
- По возможности водо- и жирорастворимые витамины должны быть добавлены в жировую эмульсию или смесь, содержащую липиды, для увеличения стабильности витаминов.

- Витамины необходимо вводить ежедневно. Жирорастворимые витамины должны вводиться одновременно с жировой эмульсией.

- Оптимальные дозы приведены в табл. 2, хотя в ряде исследований они различаются.

- Рутинный мониторинг концентраций витаминов в плазме крови во время проведения краткосрочного ПП не является обязательным (за исключением витамина D). У пациентов, находящихся на долгосрочном ПП (недели), мониторинг проводится при наличии клинических показаний.

- Недоношенные младенцы на ПП должны получать 700-1500 МЕ/кг в день (или 227-455 мкг/кг в день) витамина А, доношенные - 150-300 мкг/кг в день (или 2300 MЕ, 697 мкг/день). Если вводить парентеральную форму витамина А в водном растворе, то будут иметь место его существенные потери, поэтому следует вводить витамин в составе жировой эмульсии.

- Недоношенные младенцы на ПП должны получать 200-1000 MЕ/день (или 80-400 MЕ/кг в день) витамина D, доношенные младенцы до $12 \mathrm{Mеc} \mathrm{-} 400 \mathrm{ME} /$ день (или 40-150 МЕ/кг в день).

- У пациентов в педиатрической практике, получающих долгосрочное ПП, периодически необходимо мониторировать уровень витамина D во избежание развития дефицита. Пациентам, у которых концентрация 25(OH) витамина D в сыворотке крови $<50$ нмоль/л, необходимо дополнительное назначение витамина D. Пациентам на частичном ПП также необходим дополнительный прием добавок витамина D, который следует продолжить и после прекращения ПП.

- Суммарная доза витамина Е должна составлять для младенцев не более 11 мг/день, если используется жировая эмульсия, содержащая длинноцепочечные триглицериды и витамин Е. Для недоношенных младенцев суммарная доза витамина Е должна находиться в диапазоне 2,8-3,5 мг/кг в день, но не превышать
11 мг/день. Для правильной оценки статуса витамина Е необходимо использовать соотношение между уровнем витамина Е в сыворотке крови к общему содержанию липидов сыворотки.

च Доношенные и недоношенные младенцы на ПП должны получать 10 мкг/кг в день витамина К. Классические тесты оценки свертываемости крови могут быть использованы во время терапии витамином К, но они не являются специфическими для витамина К (непрямые показатели). Витамин К-зависимые протеины сыворотки крови могут быть полезными маркерами субклинического дефицита витамина К у пациентов группы риска.

- Недоношенные и доношенные младенцы до 12 мес, находящиеся на ПП, должны получать:

- 15-25 мг/кг в день витамина С;

- 0,35-0,50 мг/кг в день тиамина;

- 0,15-0,2 мг/кг в день рибофлавина и пиридоксина;

- 0,3 мкг/кг в день кобаламина;

- 4-6,8 мг/кг в день ниацина;

- 2,5 мг/кг в день пантотеновой кислоты;

- 5-8 мкг/кг в день биотина;

- 56 мг/кг в день фолиевой кислоты.

- Пациентам на ПП пищевые добавки железа предпочтительнее давать энтерально, чем парентерально. При краткосрочном ПП нецелесообразно рутинное назначение добавок железа. При долгосрочном ПП, когда невозможно адекватно поддерживать уровень железа в организме за счет энтерального приема, используется парентеральный путь. Парентеральные формы железа должны обеспечить поступление железа в дозе 200-250 мкг/кг в день у недоношенных младенцев и 50-100 мкг/кг в день (максимальная доза 5 мг/день) у доношенных. У пациентов на долгосрочном ПП необходимо регулярно оценивать уровни гемоглобина и ферритина с целью предотвращения дефицита или перегрузки железом.

- В состав ПП следует включить соединения меди в дозе $40 \mathrm{mr/кг} \mathrm{в} \mathrm{день} \mathrm{для} \mathrm{недоношенных} \mathrm{младенцев}$ и 20 мг/кг в день для доношенных (максимальная доза 0,5 мг в день). При этом у пациентов на длительном ПП

Таблица 2. Рекомендованные дозы жиро- и водорастворимых витаминов Аля парентерального питания доношенных и неАоношенных младенцев в день (ESPGHAN/ESPEN/ESPR/CSPEN) [33]

\begin{tabular}{|c|c|c|}
\hline Витамин & Аоза дия недоношенных детей & Аоза Аля доношенных (Ао 12 мес) \\
\hline A & 700-1500 МЕ/кг (227-455 мкг/кг) & 150-300 мкг/кг или $2300 \mathrm{ME}$ (697 мкг) \\
\hline D & 200-1000 МЕ ияи 80-400 МЕ/кГ & 400 МЕ или 40-150 МЕ/кГ \\
\hline$E$ & 2,8-3,5 мг/кг или 2,8-3,5 ME/кг & 2,8-3,5 мг/кг или 2,8-3,5 МЕ/кг \\
\hline 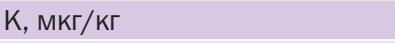 & 10 & 10 \\
\hline $\mathrm{C}, \mathrm{MKг} / \mathrm{K} \Gamma$ & $15-25$ & $15-25$ \\
\hline $\mathrm{B}_{1}, \mathrm{MK} \Gamma / \mathrm{K} \Gamma$ & $0,35-0,50$ & $0,35-0,50$ \\
\hline $\mathrm{B}_{2}, \mathrm{MK} \Gamma / \mathrm{K} \Gamma$ & $0,15-0,2$ & $0,15-0,2$ \\
\hline ПириАоксин, мкг/кг & $0,15-0,2$ & $0,15-0,2$ \\
\hline Ниацин, мкг/кг & $4-6,8$ & $4-6,8$ \\
\hline $\mathrm{B}_{12}, \mathrm{MK} \Gamma / \mathrm{K} \Gamma$ & 0,3 & 0,3 \\
\hline Пантотеновая кислота, мкг/кг & 2,5 & 2,5 \\
\hline Биотин, мкг/кг & $5-8$ & $5-8$ \\
\hline Фолиевая кислота, мкг/кг & 56 & 56 \\
\hline
\end{tabular}


Таблица 3. Потребности в железе и микроэлементах при парентеральном питании Аоношенных и недоношенных младенцев и детей до 1 года (мкг/кг в день) (ESPGHAN/ESPEN/ESPR/CSPEN) [34]

\begin{tabular}{|c|c|c|c|}
\hline \multirow[t]{2}{*}{ Микроэлемент } & \multirow{2}{*}{$\begin{array}{c}\text { Потребность } \\
\text { недоношенных } \\
\text { младенцев }\end{array}$} & \multicolumn{2}{|c|}{ Потребность Аоношенных младенцев в зависимости от возраста } \\
\hline & & 0-3 мес & 3-12 мес \\
\hline Железо & $200-250$ & $50-100$ & $50-100$ \\
\hline Цинк & $400-500$ & 250 & 100 \\
\hline MeAb & 40 & 20 & 20 \\
\hline ЙоА & $1-10$ & 1 & 1 \\
\hline Селен & 7 & $2-3$ & $2-3$ \\
\hline Марганец & $\leq 1$ & $\leq 1$ & $\leq 1$ \\
\hline Молибден & 1 & 0,25 & 0,25 \\
\hline Хром & - & - & - \\
\hline
\end{tabular}

следует мониторировать концентрацию меди в плазме крови и церулоплазмин, особенно в случаях ассоциированной с ПП болезнью печени (PNLD) и больших потерь жидкости в кишечнике.

- В состав ПП следует включить соединения цинка в дозе 400-500 мкг/кг в день у недоношенных младенцев, 250 мкг/кг в день у доношенных младенцев до 3 мес и 100 мкг/кг в день у детей от 3 до 12 мес (максимальная доза 5 мкг в день). Состояние обмена цинка (концентрация цинка в плазме, уровень щелочной фосфатазы) необходимо периодически проверять у пациентов на долгосрочном ПП, особенно с потерями жидкости в кишечнике (например, илеостома).

- В составе ПП необходимо обеспечить поступление йода в дозе 1-10 мг/кг в день у недоношенных младенцев и по крайней мере 1 мг/кг в день у доношенных. У пациентов на долгосрочном ПП необходимо мониторировать уровень тиреоидных гормонов.

- Необходимо обеспечить поступление в организм селена в дозе 7 мкг/кг в день у недоношенных младенцев и 2-3 мкг/кг в день у доношенных с максимальной дозой 100 мкг/день. Уровень селена в плазме крови необходимо регулярно мониторировать у пациентов на долгосрочном ПП и с почечной недостаточностью.

- При долгосрочном ПП следует обеспечить поступление марганца в дозе не более 1 мг/кг в день (максимум 50 мг/день). При этом необходимо регулярно мониторировать содержание марганца в крови. В случае развития холестаза нужно определить концентрацию марганца и его введение прекратить.

- При долгосрочном ПП следует обеспечить поступление молибдена в дозе 1 мг/кг в день у младенцев с низкой массой тела и 0,25 мг/кг в день у других младенцев.

\section{Современные мультивитаминные комплексы $\Delta \wedge$ п парентерального питания в неонатологии}

В настоящее время в Российской Федерации разрешены только водо- и жирорастворимые витамины для ПП новорожденных - Солувит Н и Виталипид Н детский соответственно.

Солувит Н - лиофилизированный стерильный порошок, содержаший водорастворимые витамины (биотин, натрия аскорбат, натрия пантотенат, никотинамид, пиридоксина гидрохлорид, рибофлавина натрия фосфата дигидрат, тиамина мононитрат, фолиевая кислота, цианокобаламин). Применяется как составная часть полного или частичного ПП вместе с инфузионными растворами для внутривенного введения и обеспечивает суточную потребность в водорастворимых витаминах у детей. Является единственным препаратом, содержащим все водорастворимые витамины для парентерального введения. Парентеральное введение мультивитаминных комплексов имеет следующие преимущества: высокую биодоступность, что обеспечивает максимально возможное усвоение витаминов тканями органов и быстрое включение в биохимические процессы; возможность более точного дозирования; исключение побочных эффектов со стороны ЖКТ, связанных с воздействием на слизистую оболочку.

Виталипид Н детский содержит комплекс жирорастворимых витаминов: $\alpha$-токоферол (витамин Е), ретинола пальмитат (витамин A), фитоменадион (витамин К) и эргокальциферол (витамин D). Виталипид H детский показан новорожденным и детям до 11 лет для обеспечения суточной потребности в жирорастворимых витаминах $A, D_{2}, E_{1}$ при ПП и ряде заболеваний, в патогенезе которых существенную роль играет дефицит жирорастворимых витаминов. Позволяет проводить дифференцированное лечение и профилактику гиповитаминозов жирорастворимых витаминов у всех групп новорожденных без риска перегрузки. Обладает высокой биодоступностью, способностью быстро включаться в биохимические процессы организма. Уникальность препарата обусловлена также наличием витамина $\mathrm{K}_{1}$ (единственная инъекционная форма этого витамина), обеспечивающего нормальное развитие и функционирование системы свертывания крови у детей, в том числе в критических состояниях.

Таким образом, водо-, жирорастворимые витамины и микроэлементы - обязательные компоненты всего периода парентерального питания новорожденных, необходимый компонент их успешного выхаживания. Введение микронутриентов следует начинать с первого дня парентерального питания и прекращать вместе с его окончанием. 


\section{CBEAEHИЯ ОБ АВTOPAX}

Горелик Константин Давидович (Konstantin D. Gorelik) - заведующий отделением реанимации новорожденных СПб ГБУЗ «ДГМ КСЦ ВМТ», ассистент кафедры детской хирургии с курсом реанимации ФГБОУ ВО ПСПбГМУ им. И.П. Павлова Минздрава России, Санкт-Петербург, Российская Федерация

E-mail: gorelikkd@mail.ru

https://orcid.org/0000-0003-2474-8729

Горелик Юлия Владимировна (Yulia V. Gorelik) - главный неонатолог Санкт-Петербурга, заместитель главного врача по реанимации и анестезиологии СПб ГБУз «ДГМ КСЦ ВМТ», Санкт-Петербург, Российская Федерация

E-mail: gorelikjv@mail.ru

https://orcid.org/0000-0002-9953-105X

Дмитриев Александр Владимирович (Aleksandr V. Dmitriev) - доктор медицинских наук, эксперт Северо-Западной ассоциации парентерального и энтерального питания, Санкт-Петербург, Российская Федерация

E-mail: avd.dmitriev@gmail.com

Быков Кирилл Владимирович (Kirill V. Bykov) - врач анестезиолог-реаниматолог 39-го отделения СПб ГБУ3 «ДГМ КСЦ ВМТ», Санкт-Петербург, Российская Федерация

E-mail: kvbykov92@gmail.com

https://orcid.org/0000-0002-5921-7840

\section{АИТЕРАТУРА}

1. Ерпулева Ю.В., Аекманов А.У. Основные принципы парентерального питания в пеАиатрии (рекомендации Европейского Общества Парентерального и Энтерального Питания - ESPEN) // Российский Вестник Аетской хирургии, анестезиологии и реаниматологии. 2011. № 2. С. 82-88.

2. Ерпулева Ю.В., Чубарова А.И., Вайнштейн Н.П. и Ар. Осложнения Алительного парентерального питания новорожАенных и Аетей грудного возраста // Российский Вестник детской хирургии, анестезиологии и ре аниматологии. 2017. Т. 7, № 1. С. 59-69.

3. Ерпулева Ю.В. Парентеральное питание у детей // Российский Вестник детской хирургии, анестезиологии и реаниматологии. 2018. T. 8, № 1. С. 49-56.

4. Горелик К.А., Горелик Ю.В., Амитриев А.В., Быков К.В. Жирные кислоты в составе жировых эмульсий Аля парентерального питания в неонатологии // Неонатология: новости, мнения, обучение. 2019. Т. 7, № 1. C. 54-60. DOI: 10.24411/2308-2402-2019-11007

5. Mihatsch W.A., Braegger C., Bronsky J. et al. ESPGHAN/ESPEN/ ESPR/CSPEN guidelines on pediatric parenteral nutrition // Clin. Nutr. 2018. Vol. 37. P. 2303-2305.

6. Парентеральное питание новорожденных. Клинические реко мендации. Российская Ассоциация специалистов перинатальной меди цины совместно с Ассоциацией неонатологов. 2015. С. 1-31.

7. Hanson L.A. Biology of Human Milk, Nestle Nutrition Workshop Series. New York : Raven Press, 1998.

8. Takada M., Shimada M., Hosono S. et al. Trace elements and mineral requirements for very low birth weight infants in rickets of prematurity // Early Hum. Dev. 1992. Vol. 29. P. 333-338. DOI: 10.1016/03783782(92)90188-M

9. Krachler M., Rossipal E., Micetic-Turk D. Trace element transfer from the mother to the newborn investigations on triplets of colostrum, maternal and umblical cord sera // Eur. J. Clin. Nutr. 1999. Vol. 53. P. 486-494. DOI: $10.1038 /$ sj.ejcn.1600781.

10. Özdemir H.S., Karadas F., Yörük I. et al. Vitamin and minera levels of newborns in van basin and their relation to maternal vitamin and mineral status // Open J. Pediatr. 2014. Vol. 4. P. 107-114. DOI: 10.4236/ ojped.2014.41015

11. Dallaire F., Dewailly E., Shademani R. et al. Vitamin A concentration in umbilical cord blood of infants from three separate regions of the province of Québec (Canada) // Can. J. Public Health. 2003. Vol. 94, N 5. P. 386-390.

12. Pacifici M.G. Effects of vitamin $E$ in neonates and young infants // Int. J. Pediatr. 2016. Vol. 4, N 5. P. 1745-1757.

13. De Vries J.Y., Shikha Pundir S., Mckenzie E. et al. Maternal circulating vitamin status and colostrum vitamin composition in healthy lactating women - a systematic approach // Nutrients. 2018. Vol. 10. P. 687. DOI: $10.3390 /$ nu10060687
14. Koo W.W., Tsang R.C., Succop P. et al. Minimal vitamin D and high calcium and phosphorus needs of preterm infants receiving parenteral nutrition // J. Pediatr. Gastroenterol. Nutr. 1989. Vol. 8. P. 225-233.

15. De Freitas B.A.C., Leao R.T., Gomes A.P., Siqueira-Barista R. Nutrition therapy and neonatal sepsis // Rev. Bras. Ter Intensiva. 2011 Vol. 23, N 4. P. 492-498.

16. Gamal T.S., Madiha A.A.S., Hanan M.K. et al. Neonatal and maternal 25-OH vitamin D serum levels in neonates with early-onset sepsis // Children. 2017. Vol. 4. P. 37. DOI: 10.3390/children4050037

17. Amrein K., Litonjua A.A., Moromizato T. et al. Increases in prehospitalization serum $25(\mathrm{OH})$ D concentrations are associated with improved 30-day mortality after hospital admission: a cohort study // Clin. Nutr. 2016. Vol. 35. P. 514-521.

18. Rech M.A., Hunsaker T., Rodriguez J. Deficiency in 25-hydroxy vitamin $\mathrm{D}$ and 30-day mortality in patients with severe sepsis and septic shock // Am. J. Crit. Care. 2014. Vol. 23. P. e72-e79.

19. Ginde A.A., Camargo C.A.J., Shapiro N.I. 25-OH Vitamin D insufficiency and sepsis severity in emergency department patients with suspected infection // Acad. Emerg. Med. 2011. Vol. 18. P. 551-554.

20. Phelps D.L. Current perspectives on vitamin E in infant nutrition // Am. J. Clin. Nutr. 1987. Vol. 46, N 1. Suppl. P. 187-191.

21. Brion L.P., Bell E.F., Raghuveer T.S., Soghier L. What is the appropriate intravenous dose of vitamin $E$ for very-low-birth-weight infants? // J. Perinatol. 2004. Vol. 24, N 4. P. 205-207.

22. Brion L.P., Bell E.F., Raghuveer T.S. Vitamin E supplementation for prevention of morbidity and mortality in preterm infants // Cochrane Database Syst. Rev. 2003. Vol. 4. CD003665.

23. Vogelsang A., van Lingen R.A., Slootstra J. et al. Antioxidant role of plasma carotenoids in bronchopulmonary dysplasia in preterm infants // Int. J. Vitam. Nutr. Res. 2009. Vol. 79. P. 288-296. DOI: 10.1024/0300-9831.79.56.288

24. Darlow B.A., Graham P.J. Vitamin A supplementation for preventing morbidity and mortality in very low birthweight infants // Cochrane Database Syst. Rev. 2002. Vol. 4. CD000501.

25. Darlow B.A., Graham P.J. Vitamin A supplementation to prevent mortality and short- and long-term morbidity in very low birthweight infants // Cochrane Database Syst. Rev. 2011. Vol. 10. CD000501.

26. Skouroliakou M., Konstantinou D., Koutri K. et al. A double-blind, randomized clinical trial of the effect of $w-3$ fatty acids on the oxidative stress of preterm neonates fed through parenteral nutrition // Eur. J. Clin. Nutr. 2010. Vol. 64, N 9. P. 940-947.

27. Thauvin-Robinet C., Faivre L., Barbier M.L. et al. Severe lactic acidosis and acute thiamin deficiency: a report of 11 neonates with unsupplemented total parenteral nutrition // J. Inherit. Metab. Dis. 2004. Vol. 27, N 5. P. 700-704. 
28. Xin Y., Wan D.H., Chu Q. et al. Severe sepsis as an initia presentation in children with Wernicke's encephalopathy: report of a case and literature review // Zhonghua Erke Zazhi. 2011. Vol. 49, N 8. P. 612 616.

29. Albersen M., Groenendaal F., Van der Ham M. et al. Vitamin B6 vitamin concentrations in cerebrospinal fluid differ between preterm and term newborn infants // Pediatrics. 2012. Vol. 130. P. e191-e198.

30. Bako W. et al. Short bowel syndrome in children - own experience // Med. Wieku Rozwoj. 2006. Vol. 10, N 2. P. 563-572.

31. Haiden N. et al. A randomized, controlled trial of the effects of adding vitamin B12 and folate to erythropoietin for the treatment of anemia of prematurity // Pediatrics. 2006. Vol. 118, N 1. P. 180 188.

32. Kodama H. Trace element deficiency in infants and children // JMAJ. 2004. Vol. 47, N 8. P. 376-381.

33. Bronsky J., Campoy C., Braegger. ESPGHAN/ESPEN/ESPR/CSPEN guidelines on pediatric parenteral nutrition. Vitamins // Clin. Nutr. 2018 Vol. 37, N 6. P. $2366-2678$.

34. Domellof M., Szitanyi P., Simchowitz V. et al. ESPGHAN/ESPEN/ ESPR/CSPEN guidelines on pediatric parenteral nutrition. Iron and trace minerals // Clin. Nutr. 2018. Vol. 37. P. 2354-2359. DOI: 10.1016/ j.clnu.2018.06.949

\section{REFERENCES}

1. Erpuleva Yu.V., Lekmanov A.U. Basic princips of parenteral nutrition in pediatrics (ESPEN Reacomendations). Rossiyskiy vestnik detskoy khirurgii, anesteziologii i reanimatilogii [Russian Bulletin of Pediatric Surgery, Anesthesiology and Reanimatology]. 2011; (2): 82-8. (in Russian)

2. Erpuleva Yu.V., Chubarova A.I., Vaynshtein N.P., et al. Complications of long parenteral nutrition of newbors and infants. Rossiyskiy vestnik detskoy khirurgii, anesteziologii i reanimatilogii [Russian Bulletin of Pediatric Surgery, Anesthesiology and Reanimatology]. 2017; 7 (1): 59-69. (in Russian)

3. Erpuleva Yu.V. Parenteral nutrition in childrens. Rossiyskiy vestnik detskoy khirurgii, anesteziologii i reanimatilogii [Russian Bulletin of Pediatric Surgery, Anesthesiology and Reanimatology]. 2018; 8 (1): 49-56. (in Russian)

4. Gorelik K.D., Gorelik Yu.V., Dmitriev A.V., Bykov K.V. Fatty acids as a part of lipid emulsions for parenteral nutrition in neonatology. Neonatologiya: novosti, mneniya, obuchenie [Neonatology: News, Opinions, Training]. 2019; 7 (1): 54-60. (in Russian)

5. Mihatsch W.A., Braegger C., Bronsky J. et al. ESPGHAN/ESPEN/ ESPR/CSPEN guidelines on pediatric parenteral nutrition. Clin Nutr. 2018; 37: 2303-5.

6. Newborns parenteral nutrition. Clinical recommendation. Russian association of specialists in perinatology with neonatology association. 2015: 1-31. (in Russian)

7. Hanson L.A. Biology of Human Milk, Nestle Nutrition Workshop Series. New York : Raven Press, 1998.

8. Takada M., Shimada M., Hosono S., et al. Trace elements and mineral requirements for very low birth weight infants in rickets of prematurity. Early Hum Dev. 1992; 29: 333-8. D0I: 10.1016/0378-3782(92)90188-M

9. Krachler M., Rossipal E., Micetic-Turk D. Trace element transfe from the mother to the newborn investigations on triplets of colostrum maternal and umblical cord sera. Eur J Clin Nutr. 1999; 53: 486-94. DOI: 10.1038/sj.ejcn.1600781

10. Özdemir H.S., Karadas F., Yörük I., et al. Vitamin and mineral levels of newborns in van basin and their relation to maternal vitamin and mineral status. Open J Pediatr. 2014; 4: 107-14. DOI: 10.4236/ojped.2014.41015

11. Dallaire F., Dewailly E., Shademani R., et al. Vitamin A concentration in umbilical cord blood of infants from three separate regions of the province of Québec (Canada). Can J Public Health. 2003; 94 (5): 386-90.

12. Pacifici M.G. Effects of vitamin $E$ in neonates and young infants. Int J Pediatr. 2016; 4 (5): 1745-57.

13. De Vries J.Y., Shikha Pundir S., Mckenzie E., et al. Maternal circulating vitamin status and colostrum vitamin composition in healthy lactating women - a systematic approach. Nutrients. 2018; 10: 687. DOI: 10.3390/nu10060687

14. Koo W.W., Tsang R.C., Succop P., et al. Minimal vitamin D and high calcium and phosphorus needs of preterm infants receiving parenteral nutrition. J Pediatr Gastroenterol Nutr. 1989; 8: 225-33.

15. De Freitas B.A.C., Leao R.T., Gomes A.P., Siqueira-Barista R. Nutrition therapy and neonatal sepsis. Rev Bras Ter Intensiva. 2011; 23 (4): 492-8.

16. Gamal T.S., Madiha A.A.S., Hanan M.K., et al. Neonatal and maternal $25-\mathrm{OH}$ vitamin $\mathrm{D}$ serum levels in neonates with early-onset sepsis. Children. 2017; 4: 37. DOI: 10.3390/children4050037
17. Amrein K., Litonjua A.A., Moromizato T., et al. Increases in pre-hospitalization serum $25(\mathrm{OH}) \mathrm{D}$ concentrations are associated with improved 30-day mortality after hospital admission: a cohort study. Clin Nutr. 2016; 35: 514-21.

18. Rech M.A., Hunsaker T., Rodriguez J. Deficiency in 25-hydroxy vitamin $D$ and 30-day mortality in patients with severe sepsis and septic shock. Am J Crit Care. 2014; 23: e72-9.

19. Ginde A.A., Camargo C.A.J., Shapiro N.I. 25-OH Vitamin D insufficiency and sepsis severity in emergency department patients with suspected infection. Acad Emerg Med. 2011; 18: 551-4.

20. Phelps D.L. Current perspectives on vitamin $E$ in infant nutrition. Am J Clin Nutr. 1987; 46 (1 Suppl): 187-91.

21. Brion L.P., Bell E.F., Raghuveer T.S., Soghier L. What is the appropriate intravenous dose of vitamin $\mathrm{E}$ for very-low-birth-weight infants? J Perinatol. 2004; 24 (4) 205-7.

22. Brion L.P., Bell E.F., Raghuveer T.S. Vitamin E supplementation for prevention of morbidity and mortality in preterm infants. Cochrane Database Syst Rev. 2003; 4: CD003665.

23. Vogelsang A., van Lingen R.A., Slootstra J. et al. Antioxidant role of plasma carotenoids in bronchopulmonary dysplasia in preterm infants. Int Vitam Nutr Res. 2009; 79: 288-96. DOI: 10.1024/0300-9831.79.56.288

24. Darlow B.A., Graham P.J. Vitamin A supplementation for preventing morbidity and mortality in very low birthweight infants. Cochrane Database Syst Rev. 2002; 4: CD000501.

25. Darlow B.A., Graham P.J. Vitamin A supplementation to prevent mortality and short- and long-term morbidity in very low birthweight infants. Cochrane Database Syst Rev. 2011; 10: CD000501.

26. Skouroliakou M., Konstantinou D., Koutri K., et al. A double-blind randomized clinical trial of the effect of $w-3$ fatty acids on the oxidative stress of preterm neonates fed through parenteral nutrition. Eur J Clin Nutr. 2010; 64 (9): 940-7.

27. Thauvin-Robinet C., Faivre L., Barbier M.L., et al. Severe lactic acidosis and acute thiamin deficiency: a report of 11 neonates with unsupplemented total parenteral nutrition. J Inherit Metab Dis. 2004; 27 (5): 700-4.

28. Xin Y., Wan D.H., Chu Q., et al. Severe sepsis as an initial presentation in children with Wernicke's encephalopathy: report of a case and literature review. Zhonghua Erke Zazhi. 2011; 49 (8): 612-6.

29. Albersen M., Groenendaal F., Van der Ham M., et al. Vitamin B6 vitamin concentrations in cerebrospinal fluid differ between preterm and term newborn infants. Pediatrics. 2012; 130: e191-8.

30. Bako W., et al. Short bowel syndrome in children - own experience. Med Wieku Rozwoj. 2006; 10 (2): 563-72.

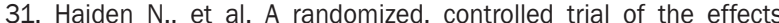
of adding vitamin B12 and folate to erythropoietin for the treatment of anemia of prematurity. Pediatrics. 2006; 118 (1): 180-8.

32. Kodama H. Trace element deficiency in infants and children. JMAJ. 2004; 47 (8): 376-81.

33. Bronsky J., Campoy C., Braegger. ESPGHAN/ESPEN/ESPR/CSPEN guidelines on pediatric parenteral nutrition. Vitamins. Clin Nutr. 2018, 37 (6): $2366-78$

34. Domellof M., Szitanyi P., Simchowitz V., et al. ESPGHAN/ESPEN/ ESPR/CSPEN guidelines on pediatric parenteral nutrition. Iron and trace minerals. Clin Nutr. 2018; 37: 2354-9. DOI: 10.1016/j.clnu.2018.06.949 